\title{
Hierarchy of magnon mode entanglement in antiferromagnets
}

\author{
Vahid Azimi Mousolou $\odot,{ }^{1,2,3, *}$ Andrey Bagrov, ${ }^{1,4}$ Anders Bergman, ${ }^{1}$ Anna Delin, ${ }^{1,5,6}$ Olle Eriksson, ${ }^{1,7}$ Yuefei Liu, ${ }^{5}$ \\ Manuel Pereiro $\odot,{ }^{1}$ Danny Thonig, ${ }^{7}$ and Erik Sjöqvist $\odot^{1, \dagger}$ \\ ${ }^{1}$ Department of Physics and Astronomy, Uppsala University, Box 516, SE-751 20 Uppsala, Sweden \\ ${ }^{2}$ Department of Applied Mathematics and Computer Science, \\ Faculty of Mathematics and Statistics, University of Isfahan, Isfahan 81746-73441, Iran \\ ${ }^{3}$ School of Mathematics, Institute for Research in Fundamental Sciences (IPM), P. O. Box 19395-5746, Tehran, Iran \\ ${ }^{4}$ Theoretical Physics and Applied Mathematics Department, Ural Federal University, Ekaterinburg 620002, Russia \\ ${ }^{5}$ Department of Applied Physics, School of Engineering Sciences, \\ KTH Royal Institute of Technology, AlbaNova University Center, SE-10691 Stockholm, Sweden \\ ${ }^{6}$ Swedish e-Science Research Center (SeRC), KTH Royal Institute of Technology, SE-10044 Stockholm, Sweden \\ ${ }^{7}$ School of Science and Technology, Örebro University, SE-701 82, Örebro, Sweden
}

(Received 4 June 2020; revised 28 October 2020; accepted 30 November 2020; published 15 December 2020)

Continuous variable entanglement between magnon modes in Heisenberg antiferromagnets with Dzyaloshinskii-Moriya (DM) interaction is examined. Different bosonic modes are identified, which allows us to establish a hierarchy of magnon entanglement. We argue that entanglement between magnon modes is determined by a simple lattice-specific parameter, together with the ratio of the strengths of the DM and Heisenberg exchange interactions, and that magnon entanglement can be detected by means of quantum homodyne techniques. As an illustration of the relevance of our findings for possible entanglement experiments in the solid state, a typical antiferromagnet with the perovskite crystal structure is considered, and it is shown that long wave length magnon modes have a maximal degree of entanglement.

DOI: 10.1103/PhysRevB.102.224418

\section{INTRODUCTION}

Quantum entanglement allows particles to act as a single nonseparable entity, no matter how far apart they are. This is the feature that was initially used in the Einstein-PodolskyRosen (EPR) argument against completeness of quantum mechanics [1]. The original form of the EPR argument is closely related to continuous variable (CV) entanglement [2-4], which describes entanglement between bosonic modes. Such systems are characterized by an infinite number of allowed states, which makes them different from the finitedimensional Hilbert spaces associated with discrete variable (e.g., qubit) systems. Nevertheless, just like discrete variable entanglement, $\mathrm{CV}$ entanglement provides an essential resource for quantum technologies, allowing for universal quantum information processing [5], quantum teleportation [5-7], quantum memories [5,8], and quantum enhanced measurement resolution [9].

It is natural to expect that quantum systems, in which information is carried in a wavelike form, in general, can show entanglement. However, the question is how clear the

\footnotetext{
*v.azimi@sci.ui.ac.ir

†erik.sjoqvist@physics.uu.se
}

Published by the American Physical Society under the terms of the Creative Commons Attribution 4.0 International license. Further distribution of this work must maintain attribution to the author(s) and the published article's title, journal citation, and DOI. Funded by Bibsam. entanglement can be demonstrated and what quantum systems might be appropriate for potential applications. In the solid state, there are several collective modes that could be suitable hosts of entanglement and provide new and unconventional resources for photon entanglement. Magnons, which are the focus of this investigation, are collective wavelike excitations of a magnet with a well-established quantum nature [10]. They can be found in an energy range of up to $\sim 500 \mathrm{meV}$, and with wavelengths spanning a range of hundreds of lattice constants to just a few. Low-energy magnon excitations can be observed in different classes of magnetic materials, such as ferromagnets, antiferromagnets, and ferrimagnets, and each class has a vast space of materials to choose from. One of the broadest classes of antiferromagnets can be found in oxide compounds, in particular, transition metal oxides [11], where for long wavelengths, the dispersion relation is essentially linear. In this paper, we focus on magnon $\mathrm{CV}$ entanglement in antiferromagnets, in which both the Heisenberg exchange and the Dzyaloshinskii-Moriya (DM) interactions may be relevant [10].

For our discussion, we note first that magnons are usually described in terms of an energy dispersion. Most experimental efforts have focused on detecting the relationship between the energy and crystal momentum. As outlined in the present paper, more information, such as magnon mode entanglement, can be extracted from the eigenvectors that describe the magnon states. Just like for photons [12], magnon states can be represented in terms of different physically natural collective modes and, for some of these, nontrivial entanglement is possible. 


\section{MAGNON MODE ENTANGLEMENTS}

\section{A. Heisenberg interaction}

To begin, we consider the quantum antiferromagnetic Heisenberg Hamiltonian on a bipartite lattice,

$$
H_{0}=J \sum_{\langle i j\rangle} \mathbf{S}_{i} \cdot \mathbf{S}_{j}, \quad J>0,
$$

where $\mathbf{S}_{i}$ is the spin- $S$ operator on site $i$ and $J$ is the strength of the exchange interaction. Using the Holstein-Primakoff transformation at low temperatures $\left(k_{B} T \ll J\right)$ followed by the Fourier transformation, one can express the spin Hamiltonian (trivial terms and zero-point energies are neglected from now on) in terms of bosonic operators as

$$
H_{0}=z_{J} J \sum_{\mathbf{k}}\left[a_{\mathbf{k}}^{\dagger} a_{\mathbf{k}}+b_{\mathbf{k}}^{\dagger} b_{\mathbf{k}}+\gamma_{\mathbf{k}} a_{\mathbf{k}} b_{\mathbf{k}}+\gamma_{-\mathbf{k}} a_{\mathbf{k}}^{\dagger} b_{\mathbf{k}}^{\dagger}\right],
$$

with the lattice specific parameter $\gamma_{\mathbf{k}}=\frac{1}{z} \sum_{\delta} e^{i \mathbf{k} \cdot \delta}, z$ being the coordination number of the lattice, and the sum over $\delta$ is carried out over nearest neighbors. Here, $a_{\mathbf{k}}^{\dagger}\left(a_{\mathbf{k}}\right)$ and $b_{\mathbf{k}}^{\dagger}\left(b_{\mathbf{k}}\right)$ are bosonic creation (annihilation) operators representing two magnon modes with wave vector $\mathbf{k}$ that are associated with the two sublattices (see Appendix A).

By employing the Bogoliubov transformation,

$$
\left(\begin{array}{c}
a_{\mathbf{k}} \\
b_{\mathbf{k}}^{\dagger}
\end{array}\right)=\left(\begin{array}{ll}
u_{\mathbf{k}} & v_{\mathbf{k}} \\
v_{\mathbf{k}}^{*} & u_{\mathbf{k}}^{*}
\end{array}\right)\left(\begin{array}{c}
\alpha_{\mathbf{k}} \\
\beta_{\mathbf{k}}^{\dagger}
\end{array}\right)
$$

with $u_{\mathbf{k}}$ and $v_{\mathbf{k}}$ given by

$$
\begin{aligned}
& \left|u_{\mathbf{k}}\right|^{2}=\frac{1}{2 \sqrt{1-\left|\gamma_{\mathbf{k}}\right|^{2}}}+\frac{1}{2}, \quad\left|v_{\mathbf{k}}\right|^{2}=\frac{1}{2 \sqrt{1-\left|\gamma_{\mathbf{k}}\right|^{2}}}-\frac{1}{2}, \\
& \frac{v_{\mathbf{k}}}{u_{\mathbf{k}}^{*}}=-\frac{1-\sqrt{1-\left|\gamma_{\mathbf{k}}\right|^{2}}}{\gamma_{\mathbf{k}}},
\end{aligned}
$$

where $\left|\gamma_{\mathbf{k}}\right|<1$, we obtain the Hamiltonian in diagonal form,

$$
H_{0}=\sum_{\mathbf{k}} \epsilon_{\mathbf{k}}\left(\alpha_{\mathbf{k}}^{\dagger} \alpha_{\mathbf{k}}+\beta_{\mathbf{k}}^{\dagger} \beta_{\mathbf{k}}\right)
$$

in terms of the new bosonic operators $(\alpha, \beta)$. For the antiferromagnetic magnon dispersion relation, we find $\epsilon_{\mathbf{k}}=$ $z S J \sqrt{\left(1-\left|\gamma_{\mathbf{k}}\right|^{2}\right)}$ (see Appendix A for derivation).

The ground state of $H_{0}$ in the $(\alpha, \beta)$ modes reads $\left|\psi_{0}\right\rangle=$ $\prod_{\mathbf{k}}\left|0 ; \alpha_{\mathbf{k}}\right\rangle\left|0 ; \beta_{\mathbf{k}}\right\rangle$, which is a separable vacuum state with vanishing entropy of entanglement [13], i.e., $E_{0}^{(\alpha, \beta)}=0$. By making the inverse Bogoliubov transformation, we may express the ground state as

$$
\left|\psi_{0}\right\rangle=\prod_{\mathbf{k}}\left|r_{\mathbf{k}}, \phi_{\mathbf{k}}\right\rangle
$$

with the two-mode generalized coherent state

$$
\left|r_{\mathbf{k}}, \phi_{\mathbf{k}}\right\rangle=\frac{1}{\cosh r_{\mathbf{k}}} \sum_{n=0}^{\infty} e^{i n \phi_{\mathbf{k}}} \tanh ^{n} r_{\mathbf{k}}\left|n ; a_{\mathbf{k}}\right\rangle\left|n ; b_{\mathbf{k}}\right\rangle
$$

in the occupation number basis $\left|n ; a_{\mathbf{k}}\right\rangle$ and $\left|n ; b_{\mathbf{k}}\right\rangle$ of the $(a, b)$ modes (see Appendix B). The parameter $r_{\mathbf{k}}$ and the phase $\phi_{\mathbf{k}}$ are given by $e^{i \phi_{\mathbf{k}}} \tanh r_{\mathbf{k}}=\frac{v_{\mathbf{k}}}{u_{\mathbf{k}}^{*}}, r_{\mathbf{k}} \equiv r_{\mathbf{k}}\left(\left|\gamma_{\mathbf{k}}\right|\right) \geqslant 0$ and $\phi_{\mathbf{k}} \equiv$ $\phi_{\mathbf{k}}\left(\gamma_{\mathbf{k}}\right)=\pi-\arg \left[\gamma_{\mathbf{k}}\right]$. The entropy of entanglement in $(a, b)$ modes, which is the von Neumann entropy of the reduced

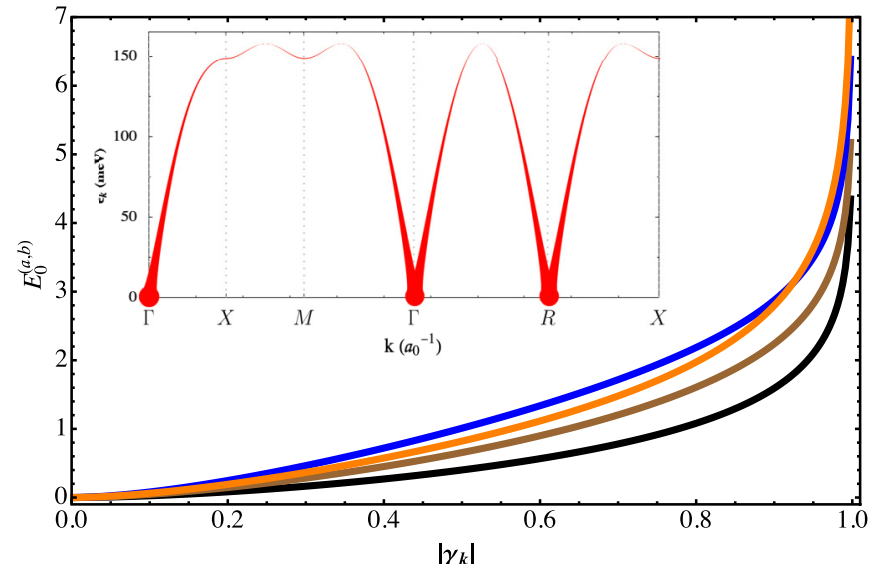

FIG. 1. The entropy of entanglement $E_{0}^{(a, b)}$ as a function of $\left|\gamma_{\mathbf{k}}\right|$ for the two-mode generalized coherent state, $\left|r_{\mathbf{k}}, \phi_{\mathbf{k}}\right\rangle$ (black curve), and for the excited states containing one magnon (brown curve), and two magnons both in one of the $\alpha$ or $\beta$ modes (blue curve) as well as two magnons each in one mode (orange curve). The inset depicts magnon dispersion of $\mathrm{SrMnO}_{3}$ for a selected path of $\mathbf{k}$ along highsymmetry directions of the BZ. The width of the curve depicts the entropy of entanglement.

density matrix of one of the two subsystems here specified by the operators $a_{\mathbf{k}}$ or $b_{\mathbf{k}}$, can be expressed as [3,13]

$$
E_{0}^{(a, b)}=\cosh ^{2} r_{\mathbf{k}} \log _{2}\left[\cosh ^{2} r_{\mathbf{k}}\right]-\sinh ^{2} r_{\mathbf{k}} \log _{2}\left[\sinh ^{2} r_{\mathbf{k}}\right] .
$$

This expression indicates that the magnon CV entanglement in the $(a, b)$ modes is, in the low-temperature regime, solely determined by the lattice geometry encoded in $\gamma_{\mathbf{k}}$. Figure 1 shows how the entropy of entanglement for the two-mode generalized coherent state varies with $\left|\gamma_{\mathbf{k}}\right|$, and in the inset we show how the energy and the entanglement depend on $\mathbf{k}$. Note that the information in the inset of Fig. 1 cannot be measured simultaneously, since the magnon dispersion is represented in $(\alpha, \beta)$ modes while the entanglement is represented by $(a, b)$ modes.

The analysis presented here is appropriate for many classes of compounds. A concrete example that is known to exhibit only nearest-neighbor Heisenberg exchange is $\mathrm{SrMnO}_{3}$ with $J=17.1 \mathrm{meV}$ [14]. The magnon dispersion of this magnetic insulator is known, both from experiments and theory, and is shown in Fig. 1 (inset), where the width of the line illustrates the entropy of entanglement as a function of $\mathbf{k}$. As is clear from the figure, when $\left|\gamma_{\mathbf{k}}\right|$ approaches 1 , the two-mode magnon entanglement becomes stronger and the entropy of entanglement formally diverges. The fact that the entanglement is largest close to the zone center is important since magnons typically are more distinct and long-lived in this regime, in comparison to the shorter wavelength magnons that have higher damping [10].

Although the main focus of our paper is on the vacuum (ground) state, we also consider magnon $\mathrm{CV}$ entanglement for excited states containing one magnon $\alpha_{\mathbf{k}}^{\dagger}\left|r_{\mathbf{k}}, \phi_{\mathbf{k}}\right\rangle$ and $\beta_{\mathbf{k}}^{\dagger}\left|r_{\mathbf{k}}, \phi_{\mathbf{k}}\right\rangle$, as well as two magnons $\left(\alpha_{\mathbf{k}}^{\dagger}\right)^{2}\left|r_{\mathbf{k}}, \phi_{\mathbf{k}}\right\rangle$, $\left(\beta_{\mathbf{k}}^{\dagger}\right)^{2}\left|r_{\mathbf{k}}, \phi_{\mathbf{k}}\right\rangle$, and $\alpha_{\mathbf{k}}^{\dagger} \beta_{\mathbf{k}}^{\dagger}\left|r_{\mathbf{k}}, \phi_{\mathbf{k}}\right\rangle$. For these states, we find that 
the entropy of entanglement behaves in a similar way as for the ground state, see Fig. 1, with the main difference that the entanglement entropy is higher for excited states.

\section{B. Dzyaloshinskii-Moriya interaction}

To further explore the material-specific features of magnon entanglement, we consider a more general spin Hamiltonian that also has DM interaction,

$$
H=H_{0}+H_{\mathrm{DM}}
$$

with $H_{\mathrm{DM}}=\sum_{\langle i j\rangle} \mathbf{D}_{i j} \cdot\left(\mathbf{S}_{i} \times \mathbf{S}_{j}\right)$ being the DM term with $\mathbf{D}_{i j}=-\mathbf{D}_{j i}$ pointing along the same fixed direction $\mathbf{D}$ for all nearest-neighbor spin pairs. By assuming $D=|\mathbf{D}|, H$ takes the form

$H=\sum_{\mathbf{k}} \epsilon_{\mathbf{k}}\left(\alpha_{\mathbf{k}}^{\dagger} \alpha_{\mathbf{k}}+\beta_{\mathbf{k}}^{\dagger} \beta_{\mathbf{k}}\right)+i z D S \sum_{\mathbf{k}}\left(\gamma_{\mathbf{k}} \alpha_{\mathbf{k}} \beta_{\mathbf{k}}-\gamma_{-\mathbf{k}} \alpha_{\mathbf{k}}^{\dagger} \beta_{\mathbf{k}}^{\dagger}\right)$,

which is not diagonal anymore in the $(\alpha, \beta)$ modes. The off-diagonal terms on the right-hand side imply that there is mixing between $\alpha$ and $\beta$ modes in the presence of DM interaction. This may cause extra magnon CV entanglement in the ground state of the system. To see this, we diagonalize $H$ by applying another Bogoliubov transformation,

$$
\left(\begin{array}{c}
\alpha_{\mathbf{k}} \\
\beta_{\mathbf{k}}^{\dagger}
\end{array}\right)=\left(\begin{array}{ll}
\eta_{\mathbf{k}} & \zeta_{\mathbf{k}} \\
\zeta_{\mathbf{k}}^{*} & \eta_{\mathbf{k}}^{*}
\end{array}\right)\left(\begin{array}{c}
\tilde{\alpha}_{\mathbf{k}} \\
\tilde{\beta}_{\mathbf{k}}^{\dagger}
\end{array}\right)
$$

where $\zeta_{\mathbf{k}}$ and $\eta_{\mathbf{k}}$ are given by the same expressions as for $v_{\mathbf{k}}$ and $u_{\mathbf{k}}$ in Eqs. (4) with $\gamma_{\mathbf{k}}$ replaced by $\Gamma_{\mathbf{k}}=\frac{i D \gamma_{\mathbf{k}}}{J \sqrt{1-\left|\gamma_{\mathbf{k}}\right|^{2}}}$ provided $\left|\Gamma_{\mathbf{k}}\right|<1$. In the $\left(\tilde{\alpha}_{\mathbf{k}}, \tilde{\beta}_{\mathbf{k}}\right)$ modes, the Hamiltonian $H$ takes the diagonal form

$$
H=\sum_{\mathbf{k}} \tilde{\epsilon}_{\mathbf{k}}\left(\tilde{\alpha}_{\mathbf{k}}^{\dagger} \tilde{\alpha}_{\mathbf{k}}+\tilde{\beta}_{\mathbf{k}}^{\dagger} \tilde{\beta}_{\mathbf{k}}\right)
$$

with the dispersion relation $\tilde{\epsilon}_{\mathbf{k}}=z S \sqrt{J^{2}\left(1-\left|\gamma_{\mathbf{k}}\right|^{2}\right)-D^{2}\left|\gamma_{\mathbf{k}}\right|^{2}}$ (see Appendix $\mathrm{C}$ for derivation).

The ground state of the diagonal Hamiltonian is a product state $|\psi\rangle=\prod_{\mathbf{k}}\left|0 ; \tilde{\alpha}_{\mathbf{k}}\right\rangle\left|0 ; \tilde{\beta}_{\mathbf{k}}\right\rangle$, where $\left|0 ; \tilde{\alpha}_{\mathbf{k}}\right\rangle$ and $\left|0 ; \tilde{\beta}_{\mathbf{k}}\right\rangle$ are vacuum states of $\tilde{\alpha}_{\mathbf{k}}$ and $\tilde{\beta}_{\mathbf{k}}$, respectively. Thus, entanglement is absent in the $(\tilde{\alpha}, \tilde{\beta})$ modes. Using the inverse transformation back into the $(\alpha, \beta)$ modes, we express the ground state as

$$
|\psi\rangle=\prod_{\mathbf{k}}\left|\tilde{r}_{\mathbf{k}}, \tilde{\phi}_{\mathbf{k}}\right\rangle,
$$

with the entangled two-mode generalized coherent state

$$
\left|\tilde{r}_{\mathbf{k}}, \tilde{\phi}_{\mathbf{k}}\right\rangle=\frac{1}{\cosh \tilde{r}_{\mathbf{k}}} \sum_{n=0}^{\infty} \tanh ^{n} \tilde{r}_{\mathbf{k}} e^{i n \tilde{\phi}_{\mathbf{k}}}\left|n ; \alpha_{\mathbf{k}}\right\rangle\left|n ; \beta_{\mathbf{k}}\right\rangle,
$$

where $\left|n ; \alpha_{\mathbf{k}}\right\rangle$ and $\left|n ; \beta_{\mathbf{k}}\right\rangle$ are the $n$th excitation of $\alpha_{\mathbf{k}}$ and $\beta_{\mathbf{k}}$, respectively. Here, $\tilde{r}_{\mathbf{k}}$ and $\tilde{\phi}_{\mathbf{k}}$ are determined by $\Gamma_{\mathbf{k}}$ in the same way as $r_{\mathbf{k}}$ and $\phi_{\mathbf{k}}$ are determined by $\gamma_{\mathbf{k}}$. In the case of $D=0$, the only relevant term is $n=0$, i.e., $\left|\tilde{r}_{\mathbf{k}}, \tilde{\phi}_{\mathbf{k}}\right\rangle=\left|0 ; \alpha_{\mathbf{k}}\right\rangle\left|0 ; \beta_{\mathbf{k}}\right\rangle$ and thus $|\psi\rangle=\left|\psi_{0}\right\rangle$. The entropy of entanglement, now specified by the $(\alpha, \beta)$ modes, becomes

$$
E^{(\alpha, \beta)}=\cosh ^{2} \tilde{r}_{\mathbf{k}} \log _{2}\left[\cosh ^{2} \tilde{r}_{\mathbf{k}}\right]-\sinh ^{2} \tilde{r}_{\mathbf{k}} \log _{2}\left[\sinh ^{2} \tilde{r}_{\mathbf{k}}\right],
$$

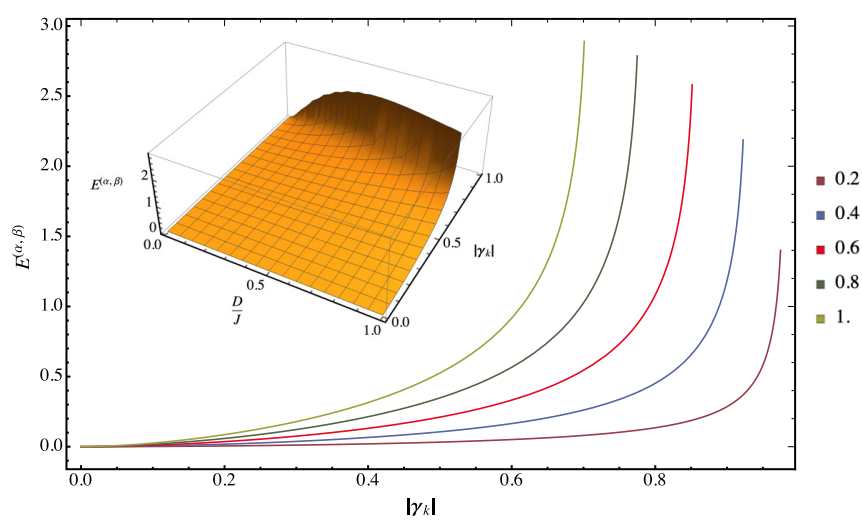

FIG. 2. The entropy of entanglement $E^{(\alpha, \beta)}$ of the two-mode generalized coherent state $\left|\tilde{r}_{\mathbf{k}}, \tilde{\phi}_{\mathbf{k}}\right\rangle$ as a function of $\left|\gamma_{\mathbf{k}}\right|$ and $\frac{D}{J}$ in the $(\alpha, \beta)$ modes. In the main figure, we show plots for different values of $\frac{D}{J}$ (that are specified by the legends), while the inset is a three-dimensional plot of the entropy of entanglement as a function of $\frac{D}{J}$ and $\left|\gamma_{\mathbf{k}}\right|$.

which is a function of $\left|\gamma_{\mathbf{k}}\right|$ and the relative coupling strength $\frac{D}{J}$. Figure 2 shows magnon CV entanglement $E^{(\alpha, \beta)}$ as a function of $\left|\gamma_{\mathbf{k}}\right|$ for selected values of $\frac{D}{J}$.

In the $(\alpha, \beta)$ modes, the two-mode magnon entanglement Eq. (15) is nontrivial, while, as shown above, the symmetric Heisenberg interaction $H_{0}$ on its own does not generate any magnon entanglement in these modes, i.e., $E_{0}^{(\alpha, \beta)}=0$. Thus, the antisymmetric DM interaction is mainly responsible for the entanglement contribution in Eq. (15), and we identify $E_{\mathrm{DM}}^{(\alpha, \beta)}=E^{(\alpha, \beta)}$ as the DM-induced entanglement.

To have a clearer picture of the hierarchy of the magnon $\mathrm{CV}$ entanglement, we transform the ground state $|\psi\rangle$ back into the original $(a, b)$ modes,

$$
|\psi\rangle=\prod_{\mathbf{k}}\left|\hat{r}_{\mathbf{k}}, \hat{\phi}_{\mathbf{k}}\right\rangle
$$

with the two-mode generalized coherent state

$$
\left|\hat{r}_{\mathbf{k}}, \hat{\phi}_{\mathbf{k}}\right\rangle=\frac{1}{\cosh \hat{r}_{\mathbf{k}}} \sum_{n=0}^{\infty} \tanh ^{n} \hat{r}_{\mathbf{k}} e^{i n \hat{\phi}_{\mathbf{k}}}\left|n ; a_{\mathbf{k}}\right\rangle\left|n ; b_{\mathbf{k}}\right\rangle,
$$

where $e^{i \hat{\phi}_{\mathbf{k}}} \tanh \hat{r}_{\mathbf{k}}=\frac{v_{\mathbf{k}} \eta_{\mathbf{k}}^{*}+u_{\mathbf{k}} \zeta_{\mathbf{k}}}{u_{\mathbf{k}}^{*} \eta_{\mathbf{k}}^{*}+v_{\mathbf{k}}^{*} \zeta_{\mathbf{k}}}, \hat{r}_{\mathbf{k}} \equiv \hat{r}_{\mathbf{k}}\left(\gamma_{\mathbf{k}}, \frac{D}{J}\right) \geqslant 0$, and $\hat{\phi}_{\mathbf{k}} \equiv$ $\hat{\phi}_{\mathbf{k}}\left(\gamma_{\mathbf{k}}, \frac{D}{J}\right)=\pi-\arg \left[\gamma_{\mathbf{k}}\left(1+i \frac{D}{J}\right)\right]$. The total entropy of entanglement in the $(a, b)$ modes for this state is

$$
E^{(a, b)}=\cosh ^{2} \hat{r}_{\mathbf{k}} \log _{2}\left[\cosh ^{2} \hat{r}_{\mathbf{k}}\right]-\sinh ^{2} \hat{r}_{\mathbf{k}} \log _{2}\left[\sinh ^{2} \hat{r}_{\mathbf{k}}\right] .
$$

Figure 3 shows the entanglement of Eq. (18) as function of $\left|\gamma_{\mathbf{k}}\right|$, for selected values of $\frac{D}{J}$. Note that increasing values of the DM interaction leads to an enhancement of the entanglement for all $\left|\gamma_{\mathbf{k}}\right|$.

Since $\left.E^{(a, b)}\right|_{D=0}=E_{0}^{(a, b)}$, we may write

$$
E^{(a, b)}=E_{0}^{(a, b)}+E_{\mathrm{DM}}^{(a, b)},
$$

where $E_{\mathrm{DM}}^{(a, b)}$ vanishes in the absence of DM interaction, and we refer to it as the DM-induced entanglement in the $(a, b)$ 


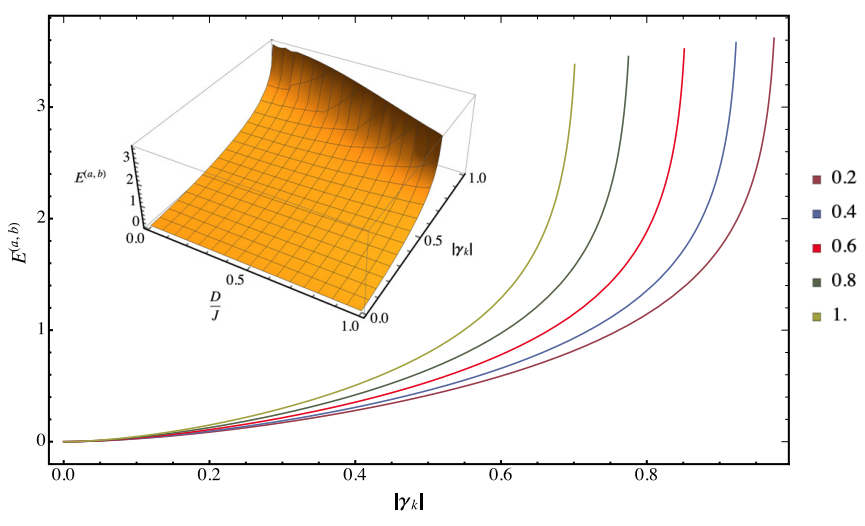

FIG. 3. The entropy of entanglement of the two-mode generalized coherent state $\left|\hat{r}_{\mathbf{k}}, \hat{\phi}_{\mathbf{k}}\right\rangle$ as a function of $\left|\gamma_{\mathbf{k}}\right|$ and the relative coupling strength $\frac{D}{J}$ in the $(a, b)$ modes. In the main figure, we show several sections of this function for different values of $\frac{D}{J}$ (that are specified by the legends), and the inset is a full three-dimensional plot.

modes. Unlike the $(\alpha, \beta)$ modes, in the $(a, b)$ modes both the Heisenberg and the DM interactions induce nonzero contributions to the magnon entanglement in the ground state.

\section{DISCUSSION AND CONCLUSIONS}

We conclude with some final remarks. In the analysis of different bosonic modes, we notice different types of twomode magnon entanglement residing in the ground state. In Fig. 4, we compare entropies of entanglement for antiferromagnets, which are effectively described by nearest-neighbor Heisenberg exchange as well as DM interaction with typical ratio $D / J \approx 30 \%$ [15-17]. It can be seen that from $(a, b)$ modes to $(\alpha, \beta)$ modes, the Heisenberg contribution to entanglement decreases while the DM-induced magnon CV entanglement increases. This is due to the fact that different bosonic modes represent different tensor product structures

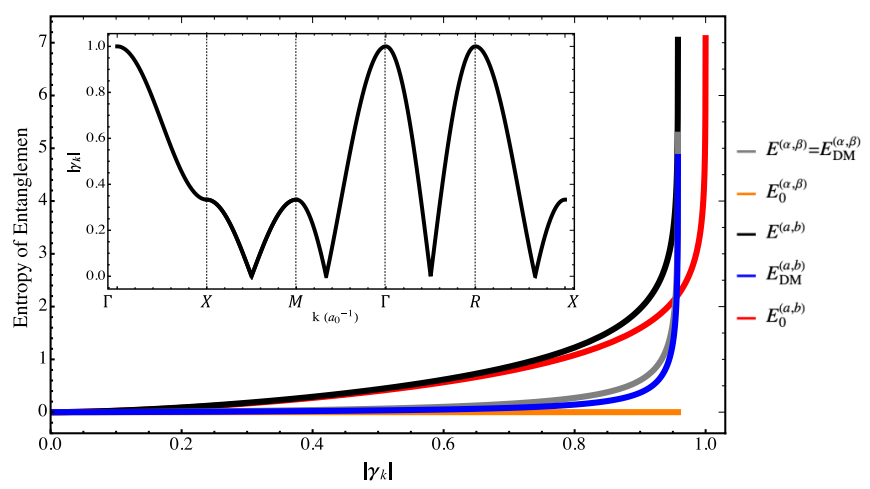

FIG. 4. Hierarchy of magnon entanglement for an antiferromagnet as a function of $\left|\gamma_{\mathbf{k}}\right|$. Definition of the different entanglement entropies is given in the main text and specified by the legends. An effective DM interaction with the strength of $30 \%$ of the Heisenberg exchange $J$ is used. To verify the hierarchy for a specific crystal structure, it is necessary to compute $\left|\gamma_{\mathbf{k}}\right|$ at different points in the BZ. To this end, the inset shows $\left|\gamma_{\mathbf{k}}\right|$ as a function of $\mathbf{k}$ in a simple cubic lattice as an example. of the Hilbert space [18]. While the $(a, b)$ modes describe naturally identifiable magnon modes being associated with each sublattice, the $(\alpha, \beta)$ and $(\tilde{\alpha}, \tilde{\beta})$ modes, which are relevant for the spectrum of $H_{0}$ and $H_{0}+H_{\mathrm{DM}}$, respectively, are hybridized and their number states are described by superpositions of excitations in the $(a, b)$ modes [19]. In this context, it should be stressed that the bosonic modes that diagonalize the spin Hamiltonian are not the same as those describing any of the nontrivially entangled magnon modes discussed here. This implies that magnon mode entanglement and magnon energy dispersion cannot be detected in the same experimental setup.

The condition for diagonalizing the Hamiltonians in terms of bosonic operators is that $\left|\gamma_{\mathbf{k}}\right|<1$ in the pure Heisenberg case and $\left|\gamma_{\mathbf{k}}\right|^{2}<\frac{J^{2}}{J^{2}+D^{2}}$ in the presence of DM interaction. Since $D$ is typically less than a few tenths of $J$ for most materials [15-17], this condition is satisfied almost everywhere in the BZ except only small parts, such as a region around zone center. We would like to remark that the entire BZ may be included in this analysis by considering a Hamiltonian that possesses single ion uniaxial anisotropy, $-\mathcal{K}(\mathbf{n} \cdot \mathbf{S})^{2}$, e.g., with easy-axis $\mathbf{n}$ along the direction of the DM vector, as long as $\left|1+\frac{2 \mathcal{K}}{z J}\right|>\sqrt{1+\frac{D^{2}}{J^{2}}}$. Note that any change of symmetry in the Hamiltonian introduces new magnon modes and hence new levels of entanglement contribution in the hierarchy of two-mode magnon entanglement. Our general message is not changed by this, although the technical level of calculations may become more intricate. It is interesting to note that already very mild uniaxial anisotropy of $0.001 \%$ of $J$ along $z$ axis, when included in a pure Heisenberg Hamiltonian $(D=0)$, allows us to regularize the magnon $\mathrm{CV}$ entanglement dependence on $\left|\gamma_{\mathbf{k}}\right|$. At $\left|\gamma_{\mathbf{k}}\right|=1$, we obtain $E_{0}^{(a, b)} \approx 9.04$. In a more generic case of Heisenberg-DMI with $D / J=0.1, z=6$, and uniaxial anisotropy of $\mathcal{K} / J=0.015$ along $\mathbf{D}$, we find $E^{(a, b)} \approx 8.094$ and $E^{(\alpha, \beta)} \approx 4.766$ at $\left|\gamma_{\mathbf{k}}\right|=1$.

The magnon $\mathrm{CV}$ entanglement is an intrinsic property of antiferromagnets, shown here to depend on the geometry of the spin lattice as encoded in $\gamma_{\mathbf{k}}$ and on the relative coupling strength $\frac{D}{J}$. Both parameters are material dependent and can vary strongly from system to system. This opens an interesting route to search for suitable entanglement hosts among the existing thousands of magnetic compounds, and poses a natural question of how magnon entanglement can be detected in an experiment. A relevant experimentally accessible quantity is the arithmetic mean of the squared quadrature variances which, in the $(a, b)$ modes, is given by

$$
\begin{aligned}
\Delta\left(\hat{r}_{\mathbf{k}}, \hat{\phi}_{\mathbf{k}}\right) & =\frac{1}{2}\left[\operatorname{Var}_{\hat{r}_{\mathbf{k}}, \hat{\phi}_{\mathbf{k}}}^{2}\left(X_{\mathbf{k}}^{A}-X_{\mathbf{k}}^{B}\right)+\operatorname{Var}_{\hat{r}_{\mathbf{k}}, \hat{\phi}_{\mathbf{k}}}^{2}\left(P_{\mathbf{k}}^{A}+P_{\mathbf{k}}^{B}\right)\right] \\
& =\cosh 2 \hat{r}_{\mathbf{k}}-\sinh 2 \hat{r}_{\mathbf{k}} \cos \hat{\phi}_{\mathbf{k}} .
\end{aligned}
$$

Here, $X_{\mathbf{k}}^{A}\left(X_{\mathbf{k}}^{B}\right)$ and $P_{\mathbf{k}}^{A}\left(P_{\mathbf{k}}^{B}\right)$ are the dimensionless position and momentum quadratures of the $a_{\mathbf{k}}\left(b_{\mathbf{k}}\right)$ mode [20], and $\operatorname{Var}_{\hat{r}_{\mathbf{k}}, \hat{\phi}_{\mathbf{k}}}(V)$ is the variance of a given Hermitian operator $V$ with respect to the state $\left|\hat{r}_{\mathbf{k}}, \hat{\phi}_{\mathbf{k}}\right\rangle$.

The parameter $\Delta\left(\hat{r}_{\mathbf{k}}, \hat{\phi}_{\mathbf{k}}\right)$ can be accessed experimentally by detecting coherences of quantum fields, the quadratures, with quantum homodyne detection techniques [2,21$23]$ adapted to magnon-photon coupling [24,25]. For instance, as a proof of principle, consider a magnon-photon coupling 
in antiferromagnetic material, as described by the Hamiltonian $H_{\mathbf{k}}^{\mathrm{m}-\mathrm{p}}=\lambda(t)\left(a_{\mathbf{k}}^{\dagger} c_{\mathbf{k}}+a_{\mathbf{k}} c_{\mathbf{k}}^{\dagger}+b_{\mathbf{k}}^{\dagger} d_{\mathbf{k}}+b_{\mathbf{k}} d_{\mathbf{k}}^{\dagger}\right)$ for a given wave vector $\mathbf{k}$. Here $(a, b)$ magnon modes interact with $(c, d)$ photon modes with time-dependent coupling strength $\lambda(t)$ describing a finite time interaction, corresponding, e.g., to a pair of short synchronized laser pulses that scatter on the material. In this way, magnon entanglement can be transferred to photon entanglement, i.e., the former can be used as a resource for the latter and, conversely, the $(a, b)$ magnon quadratures can be measured through quadratures of the $(c, d)$ modes of the scattered photons. Similar magnon-photon coupling scenarios can be considered in $(\alpha, \beta)$ as well as $(\tilde{\alpha}, \tilde{\beta})$ modes, which induce different entanglement between scattered photons.

The analysis put forth here is appropriate for many classes of compounds, but we would like to mention, in particular, the transition-metal oxides that have a vast crystallographic phase space, which allows both for tunability of $\frac{D}{J}$ as well as $\gamma_{\mathbf{k}}$. A concrete example that is known to exhibit only nearestneighbor Heisenberg exchange is $\mathrm{SrMnO}_{3}$ [14]. We also note that materials like $\mathrm{La}_{2} \mathrm{CuO}_{4}$ [26], $\mathrm{FeBO}_{3}$, and $\mathrm{CoCO}_{3}$ [27] are well-studied antiferromagnets that are known to have DM interaction in the here studied range of $\frac{D}{J}$.

Last but not least, we would like to point out that the present paper differs from previous efforts in this field, e.g., the study published in Ref. [19], where experiments of photoinduced spin dynamics were interpreted in terms of entanglement between a pair of magnon modes. Furthermore, in Refs. [28-31], investigations involved the steady-state limit and entanglement between magnon modes that were in contact with other degrees of freedom, like cavity and lattice vibrational fields. In contrast, our analysis shows that even in the absence of any external field or extra degrees of freedom, there naturally is a hierarchy of different types of two-mode magnon entanglement in eigenstates of an antiferromagnet, where each level of this hierarchy is specified by the geometry of the spin lattice and individual exchange couplings.

\section{ACKNOWLEDGMENTS}

The authors acknowledge financial support from Knut and Alice Wallenberg Foundation through Grant No. 2018.0060. V.A.M. acknowledges support from IPM through Grant No. 98810042. A.B. acknowledges financial support from the Russian Science Foundation through Grant No. 18-12-00185. A.D. acknowledges financial support from the Swedish Research Council (VR) through Grants No. 2015-04608, No. 2016-05980, and No. 2019-05304. O.E. acknowledges support from eSSENCE, SNIC and the Swedish Research Council (VR). D.T. acknowledges support from the Swedish Research Council (VR) through Grant No. 2019-03666. E.S. acknowledges financial support from the Swedish Research Council (VR) through Grant No. 2017-03832. Some of the computations were performed on resources provided by the Swedish National Infrastructure for Computing (SNIC) at the National Supercomputer Center (NSC), Linköping University, the PDC Centre for High Performance Computing (PDC-HPC), KTH, and the High Performance Computing Center North (HPC2N), Umeå University.

\section{APPENDIX A: DERIVATION OF EQS. (4) AND (5)}

Consider the nearest-neighbor antiferromagnetic Heisenberg model,

$$
H_{0}=J \sum_{\langle i j\rangle} \mathbf{S}_{i} \cdot \mathbf{S}_{j}, \quad J>0,
$$

whose spins reside on two sublattices (see Fig. 5). We use $i \in A$ and $j \in B$ notation for sites belonging to different sublattices.

Using the Holstein-Primakoff transformation, one can map the above spin Hamiltonian onto a bosonic system. On each of the sublattices, we define

$$
\begin{aligned}
& \text { Sublattice } A:\left\{\begin{array}{l}
S_{i}^{z}=S-a_{i}^{\dagger} a_{i}, \\
S_{i}^{+}=\left(2 S-a_{i}^{\dagger} a_{i}\right)^{\frac{1}{2}} a_{i} \\
S_{i}^{-}=a_{i}^{\dagger}\left(2 S-a_{i}^{\dagger} a_{i}\right)^{\frac{1}{2}},
\end{array}\right. \\
& \text { Sublattice } B:\left\{\begin{array}{l}
S_{j}^{z}=b_{j}^{\dagger} b_{j}-S \\
S_{j}^{+}=b_{j}^{\dagger}\left(2 S-b_{j}^{\dagger} b_{j}\right)^{\frac{1}{2}} \\
S_{j}^{-}=\left(2 S-b_{j}^{\dagger} b_{j}\right)^{\frac{1}{2}} b_{j},
\end{array}\right.
\end{aligned}
$$

where $a_{i}^{\dagger}\left(a_{i}\right)$ and $b_{j}^{\dagger}\left(b_{j}\right)$ are bosonic creation (annihilation) operators on sites $i \in A$ and $j \in B$, respectively. The bosonic operators of the two sublattices mutually commute.

At low temperatures $\left(k_{B} T \ll J\right)$, we have $\left\langle a_{i}^{\dagger} a_{i}\right\rangle \ll S$ and $\left\langle b_{j}^{\dagger} b_{j}\right\rangle \ll S$, which allows us to rewrite $H_{0}$ as

$$
\begin{aligned}
H_{0}= & -\frac{N z J S^{2}}{2}+z J S\left(\sum_{i \in A} a_{i}^{\dagger} a_{i}+\sum_{j \in B} b_{j}^{\dagger} b_{j}\right) \\
& +J S\left(\sum_{\langle i j\rangle} a_{i} b_{j}+a_{i}^{\dagger} b_{j}^{\dagger}\right),
\end{aligned}
$$

within linear approximation. Here, $N$ is the number of sites and $z$ is the coordination number of the lattice. We further

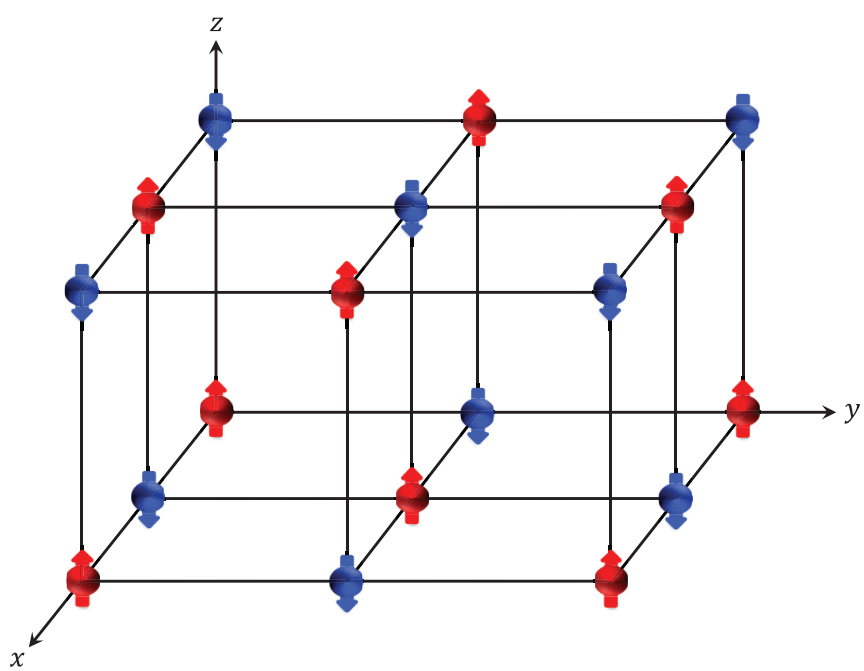

FIG. 5. Simple cubic lattice. Red and blue dots represent two sublattices with antiparallel orientation of the magnetic moment. The figure illustrates the reference frame used to indicate the direction of the DM vector, i.e., along the $z$ axis. 
perform the Fourier transformation,

$$
\begin{aligned}
a_{i} & =\sqrt{\frac{2}{N}} \sum_{\mathbf{k}} e^{-i \mathbf{k} \cdot \mathbf{r}_{i}} a_{\mathbf{k}} \Leftrightarrow a_{\mathbf{k}}=\sqrt{\frac{2}{N}} \sum_{i} e^{i \mathbf{k} \cdot \mathbf{r}_{i}} a_{i}, \\
b_{j} & =\sqrt{\frac{2}{N}} \sum_{\mathbf{k}^{\prime}} e^{i \mathbf{k}^{\prime} \cdot \mathbf{r}_{j}} b_{\mathbf{k}^{\prime}} \Leftrightarrow b_{\mathbf{k}^{\prime}}=\sqrt{\frac{2}{N}} \sum_{j} e^{-i \mathbf{k}^{\prime} \cdot \mathbf{r}_{j}} b_{j},
\end{aligned}
$$

with orthogonality relations

$$
\sum_{i} e^{ \pm i\left(\mathbf{k}-\mathbf{k}^{\prime}\right) \cdot \mathbf{r}_{i}}=\frac{N}{2} \delta_{\mathbf{k} \mathbf{k}^{\prime}}, \quad \sum_{\mathbf{k}} e^{ \pm i \mathbf{k} \cdot\left(\mathbf{r}_{j}-\mathbf{r}_{i}\right)}=\frac{N}{2} \delta_{i j},
$$

to rewrite the Hamiltonian in $\mathbf{k}$ space as

$H_{0}=-\frac{N z J S^{2}}{2}+z J S \sum_{\mathbf{k}}\left[a_{\mathbf{k}}^{\dagger} a_{\mathbf{k}}+b_{\mathbf{k}}^{\dagger} b_{\mathbf{k}}+\gamma_{\mathbf{k}} a_{\mathbf{k}} b_{\mathbf{k}}+\gamma_{-\mathbf{k}} a_{\mathbf{k}}^{\dagger} b_{\mathbf{k}}^{\dagger}\right]$.

Here

$$
\gamma_{\mathbf{k}}=\frac{1}{z} \sum_{\delta} e^{i \mathbf{k} \cdot \delta}
$$

where the sum is carried out over the vectors $\delta$ connecting a magnetic site to its nearest neighbors on the opposite sublattice.

The Hamiltonian $H_{0}$ is then diagonalized by performing the Bogoliubov transformation,

$$
\left(\begin{array}{c}
a_{\mathbf{k}} \\
b_{\mathbf{k}}^{\dagger}
\end{array}\right)=\left(\begin{array}{cc}
u_{\mathbf{k}} & v_{\mathbf{k}} \\
v_{\mathbf{k}}^{*} & u_{\mathbf{k}}^{*}
\end{array}\right)\left(\begin{array}{c}
\alpha_{\mathbf{k}} \\
\beta_{\mathbf{k}}^{\dagger}
\end{array}\right),
$$

where $u_{\mathbf{k}}$ and $v_{\mathbf{k}}$ satisfy $\left|u_{\mathbf{k}}\right|^{2}-\left|v_{\mathbf{k}}\right|^{2}=1$. The constraint assures that $\alpha_{\mathbf{k}}$ and $\beta_{\mathbf{k}}$ are bosonic operators for all $\mathbf{k}$. In the $\alpha, \beta$ representation, the Hamiltonian takes the form

$$
\begin{aligned}
H_{0}= & -\frac{N z J S(S+1)}{2}+\sum_{\mathbf{k}} \epsilon_{\mathbf{k}}\left(\alpha_{\mathbf{k}}^{\dagger} \alpha_{\mathbf{k}}+\beta_{\mathbf{k}}^{\dagger} \beta_{\mathbf{k}}+I\right) \\
& +\sum_{\mathbf{k}}\left(o_{\mathbf{k}} \alpha_{\mathbf{k}} \beta_{\mathbf{k}}+o_{\mathbf{k}}^{*} \alpha_{\mathbf{k}}^{\dagger} \beta_{\mathbf{k}}^{\dagger}\right),
\end{aligned}
$$

where

$$
\begin{aligned}
& \epsilon_{\mathbf{k}}=z J S\left(2 \operatorname{Re}\left[\gamma_{\mathbf{k}} u_{\mathbf{k}} v_{\mathbf{k}}\right]+\left|u_{\mathbf{k}}\right|^{2}+\left|v_{\mathbf{k}}\right|^{2}\right), \\
& o_{\mathbf{k}}=z J S\left(2 u_{\mathbf{k}} v_{\mathbf{k}}^{*}+\gamma_{\mathbf{k}} u_{\mathbf{k}}^{2}+\gamma_{-\mathbf{k}} v_{\mathbf{k}}^{* 2}\right) .
\end{aligned}
$$

We eliminate $o_{\mathbf{k}}$ by requiring

$$
\begin{aligned}
\left|u_{\mathbf{k}}\right|^{2} & =\frac{1}{2 \sqrt{1-\left|\gamma_{\mathbf{k}}\right|^{2}}}+\frac{1}{2}, \quad\left|v_{\mathbf{k}}\right|^{2}=\frac{1}{2 \sqrt{1-\left|\gamma_{\mathbf{k}}\right|^{2}}}-\frac{1}{2}, \\
\frac{v_{\mathbf{k}}}{u_{\mathbf{k}}^{*}} & =-\frac{1-\sqrt{1-\left|\gamma_{\mathbf{k}}\right|^{2}}}{\gamma_{\mathbf{k}}},
\end{aligned}
$$

provided $\left|\gamma_{\mathbf{k}}\right|<1$. This way, we end up with the diagonal Hamiltonian,

$$
H_{0}=-\frac{N z J S(S+1)}{2}+\sum_{\mathbf{k}} \epsilon_{\mathbf{k}}\left(\alpha_{\mathbf{k}}^{\dagger} \alpha_{\mathbf{k}}+\beta_{\mathbf{k}}^{\dagger} \beta_{\mathbf{k}}+I\right),
$$

with the antiferromagnetic magnon dispersion relation $\epsilon_{\mathbf{k}}=$ $z S \sqrt{J^{2}\left(1-\left|\gamma_{\mathbf{k}}\right|^{2}\right)}$. Equation (5) is obtained by omitting the trivial terms $-\frac{N Z J S(S+1)}{2}$ and $\sum_{\mathbf{k}} \epsilon_{\mathbf{k}} I$ of Eq. (A11).

\section{APPENDIX B: DERIVATION OF EQ. (7)}

From the diagonal expression in Eq. (A11), the ground state of the Hamiltonian $H_{0}$ in the $\alpha, \beta$ modes reads

$$
\left|\psi_{0}\right\rangle=\prod_{\mathbf{k}}\left|0 ; \alpha_{\mathbf{k}}\right\rangle\left|0 ; \beta_{\mathbf{k}}\right\rangle
$$

where $\left|0 ; \alpha_{\mathbf{k}}\right\rangle$ and $\left|0 ; \beta_{\mathbf{k}}\right\rangle$ are vacuum states of $\alpha_{\mathbf{k}}$ and $\beta_{\mathbf{k}}$, respectively, i.e.,

$$
\alpha_{\mathbf{k}}\left(\left|0 ; \alpha_{\mathbf{k}}\right\rangle\left|0 ; \beta_{-\mathbf{k}}\right\rangle\right)=\beta_{\mathbf{k}}\left(\left|0 ; \alpha_{\mathbf{k}}\right\rangle\left|0 ; \beta_{\mathbf{k}}\right\rangle\right)=0 .
$$

By expressing the product vacuum state as a linear combination of the $n$th excitations $\left|n ; a_{\mathbf{k}}\right\rangle$ and $\left|n ; b_{\mathbf{k}}\right\rangle$ of $a_{\mathbf{k}}$ and $b_{\mathbf{k}}$, respectively, i.e.,

$$
\left|0 ; \alpha_{\mathbf{k}}\right\rangle\left|0 ; \beta_{\mathbf{k}}\right\rangle=\sum_{n=0}^{\infty} c_{n ; \mathbf{k}}\left|n ; a_{\mathbf{k}}\right\rangle\left|n ; b_{\mathbf{k}}\right\rangle,
$$

and inserting it into Eq. (B2), we find

$$
c_{n+1 ; \mathbf{k}}=\frac{v_{\mathbf{k}}}{u_{\mathbf{k}}^{*}} c_{n ; \mathbf{k}} .
$$

By solving this recurrence equation with normalization constraint $\sum_{n=0}^{\infty}\left|c_{n ; \mathbf{k}}\right|^{2}=1$, the wave function amplitudes in the superposed coherent state of Eq. (B3) become

$$
c_{n ; \mathbf{k}}=\frac{e^{i n \phi_{\mathbf{k}}}}{\cosh r_{\mathbf{k}}} \tanh ^{n} r_{\mathbf{k}},
$$

where $e^{i \phi_{\mathbf{k}}} \tanh r_{\mathbf{k}}=\frac{v_{\mathbf{k}}}{u_{\mathbf{k}}^{*}}$ with

$$
\begin{aligned}
& r_{\mathbf{k}} \equiv r_{\mathbf{k}}\left(\gamma_{\mathbf{k}}\right)=\tanh ^{-1}\left(\frac{1-\sqrt{1-\left|\gamma_{\mathbf{k}}\right|^{2}}}{1+\sqrt{1-\left|\gamma_{\mathbf{k}}\right|^{2}}}\right)^{\frac{1}{2}} \geqslant 0, \\
& \phi_{\mathbf{k}} \equiv \phi_{\mathbf{k}}\left(\gamma_{\mathbf{k}}\right)=\pi-\arg \left[\gamma_{\mathbf{k}}\right] .
\end{aligned}
$$

Therefore, in the $a, b$ modes, the ground state represents a product of pairwise entangled magnetic modes

$$
\left|\psi_{0}\right\rangle=\prod_{\mathbf{k}}\left|r_{\mathbf{k}}, \phi_{\mathbf{k}}\right\rangle
$$

where

$$
\left|r_{\mathbf{k}}, \phi_{\mathbf{k}}\right\rangle=\frac{1}{\cosh r_{\mathbf{k}}} \sum_{n=0}^{\infty} e^{i n \phi_{\mathbf{k}}} \tanh ^{n} r_{\mathbf{k}}\left|n ; a_{\mathbf{k}}\right\rangle\left|n ; b_{\mathbf{k}}\right\rangle .
$$

For $\tanh r_{\mathbf{k}}<\cos \left(\phi_{\mathbf{k}}\right),\left|r_{\mathbf{k}}, \phi_{\mathbf{k}}\right\rangle$ is a two-mode squeezed state with squeezing parameter $r_{\mathbf{k}}$. Note that we have, in fact, performed the inverse of the transformation in Eq. (A7) to arrive at Eq. (B8). Similar inverse transformation techniques are applied to derive Eqs. (14) and (17) in the paper.

\section{APPENDIX C: DERIVATION OF EQ. (12)}

Consider the Hamiltonian

$$
H=H_{0}+H_{\mathrm{DM}},
$$


with DM spin-orbit interaction

$$
H_{\mathrm{DM}}=\sum_{\langle i j\rangle} \mathbf{D}_{i j} \cdot\left(\mathbf{S}_{i} \times \mathbf{S}_{j}\right),
$$

where $\mathbf{D}_{i j}=-\mathbf{D}_{j i}$ points along the same fixed direction $\mathbf{D}$ for all nearest-neighbor spin pairs. Since the Heisenberg term $H_{0}$ has spherical symmetry, without loss of generality we assume the uniform DM direction to be along the $z$ axis (see Fig. 5). Transforming the Hamiltonian $H$ into the $\alpha, \beta$ modes, we obtain

$$
\begin{aligned}
H= & -\frac{N z J S(S+1)}{2}+\sum_{\mathbf{k}} \epsilon_{\mathbf{k}}\left(\alpha_{\mathbf{k}}^{\dagger} \alpha_{\mathbf{k}}+\beta_{\mathbf{k}}^{\dagger} \beta_{\mathbf{k}}+I\right) \\
& +i z D S \sum_{\mathbf{k}}\left(\gamma_{\mathbf{k}} \alpha_{\mathbf{k}} \beta_{\mathbf{k}}-\gamma_{-\mathbf{k}} \alpha_{\mathbf{k}}^{\dagger} \beta_{\mathbf{k}}^{\dagger}\right),
\end{aligned}
$$

where we take $u_{\mathbf{k}}$ in Eq. (A10) to be real. By applying the Bogoliubov transformation

$$
\left(\begin{array}{c}
\alpha_{\mathbf{k}} \\
\beta_{\mathbf{k}}^{\dagger}
\end{array}\right)=\left(\begin{array}{ll}
\eta_{\mathbf{k}} & \zeta_{\mathbf{k}} \\
\zeta_{\mathbf{k}}^{*} & \eta_{\mathbf{k}}^{*}
\end{array}\right)\left(\begin{array}{c}
\tilde{\alpha}_{\mathbf{k}} \\
\tilde{\beta}_{\mathbf{k}}^{\dagger}
\end{array}\right)
$$

with the bosonic constraint $\left|\eta_{\mathbf{k}}\right|^{2}-\left|\zeta_{\mathbf{k}}\right|^{2}=1$, the Hamiltonian $H$ can be re-expressed in the $\tilde{\alpha}, \tilde{\beta}$ bosonic modes as

$$
\begin{aligned}
H= & -\frac{N z J S(S+1)}{2}+\sum_{\mathbf{k}} \tilde{\epsilon}_{\mathbf{k}}\left(\tilde{\alpha}_{\mathbf{k}}^{\dagger} \tilde{\alpha}_{\mathbf{k}}+\tilde{\beta}_{\mathbf{k}}^{\dagger} \tilde{\beta}_{\mathbf{k}}+I\right) \\
& +\sum_{\mathbf{k}}\left(\tilde{o}_{\mathbf{k}} \tilde{\alpha}_{\mathbf{k}} \tilde{\beta}_{\mathbf{k}}+\tilde{o}_{\mathbf{k}}^{*} \tilde{\alpha}_{\mathbf{k}}^{\dagger} \tilde{\beta}_{\mathbf{k}}^{\dagger}\right),
\end{aligned}
$$

where

$$
\begin{aligned}
& \tilde{\epsilon}_{\mathbf{k}}=z S\left[J \sqrt{1-\left|\gamma_{\mathbf{k}}\right|^{2}}\left(\left|\eta_{\mathbf{k}}\right|^{2}+\left|\zeta_{\mathbf{k}}\right|^{2}\right)-2 D \operatorname{Im}\left[\gamma_{\mathbf{k}} \eta_{\mathbf{k}} \zeta_{\mathbf{k}}\right]\right], \\
& \tilde{o}_{\mathbf{k}}=z S\left[2 J \eta_{\mathbf{k}} \zeta_{\mathbf{k}}^{*} \sqrt{1-\left|\gamma_{\mathbf{k}}\right|^{2}}+i D\left(\gamma_{\mathbf{k}} \eta_{\mathbf{k}}^{2}-\gamma_{-\mathbf{k}} \zeta_{\mathbf{k}}^{* 2}\right)\right] .
\end{aligned}
$$

Solving the equation $\tilde{o}_{\mathbf{k}}=0$ for $\eta_{\mathbf{k}}, \zeta_{\mathbf{k}}$ with $\left|\eta_{\mathbf{k}}\right|^{2}-\left|\zeta_{\mathbf{k}}\right|^{2}=1$ constraint, we find

$$
\begin{aligned}
\left|\eta_{\mathbf{k}}\right|^{2} & =\frac{1}{2 \sqrt{1-\left|\Gamma_{\mathbf{k}}\right|^{2}}}+\frac{1}{2}, \quad\left|\zeta_{\mathbf{k}}\right|^{2}=\frac{1}{2 \sqrt{1-\left|\Gamma_{\mathbf{k}}\right|^{2}}}-\frac{1}{2}, \\
\frac{\zeta_{\mathbf{k}}}{\eta_{\mathbf{k}}^{*}} & =-\frac{1-\sqrt{1-\left|\Gamma_{\mathbf{k}}\right|^{2}}}{\Gamma_{\mathbf{k}}}
\end{aligned}
$$

provided $\quad \Gamma_{\mathbf{k}}=\frac{i D \gamma_{\mathbf{k}}}{J \sqrt{1-\left|\gamma_{\mathbf{k}}\right|^{2}}} \quad$ with $\quad\left|\Gamma_{\mathbf{k}}\right|<1$. By inserting Eqs. (C7) into Eqs. (C6), we obtain the Hamiltonian $H$ in the following diagonal form:

$$
H=-\frac{N z J S(S+1)}{2}+\sum_{\mathbf{k}} \tilde{\epsilon}_{\mathbf{k}}\left(\tilde{\alpha}_{\mathbf{k}}^{\dagger} \tilde{\alpha}_{\mathbf{k}}+\tilde{\beta}_{\mathbf{k}}^{\dagger} \tilde{\beta}_{\mathbf{k}}+I\right)
$$

with dispersion relation $\tilde{\epsilon}_{\mathbf{k}}=z S \sqrt{J^{2}\left(1-\left|\gamma_{\mathbf{k}}\right|^{2}\right)-D^{2}\left|\gamma_{\mathbf{k}}\right|^{2}}$. Equation (12) is obtained by omitting the trivial terms $-\frac{N z J S(S+1)}{2}$ and $\sum_{\mathbf{k}} \tilde{\epsilon}_{\mathbf{k}} I$ of Eq. (C8).
[1] A. Einstein, B. Podolsky, and N. Rosen, Can quantummechanical description of physical reality be considered complete? Phys. Rev. 47, 777 (1935).

[2] Z. Y. Ou, S. F. Pereira, H. J. Kimble, and K. C. Peng, Realization of the Einstein-Podolsky-Rosen Paradox for Continuous Variables, Phys. Rev. Lett. 68, 3663 (1992).

[3] G. Giedke, M. M. Wolf, O. Krüger, R. F. Werner, and J. I. Cirac, Entanglement of Formation for Symmetric Gaussian States, Phys. Rev. Lett. 91, 107901 (2003).

[4] M. D. Reid, P. D. Drummond, W. P. Bowen, E. G. Cavalcanti, P. K. Lam, H. A. Bachor, U. L. Andersen, and G. Leuchs, The Einstein-Podolsky-Rosen paradox: From concepts to applications, Rev. Mod. Phys. 81, 1727 (2009).

[5] S. L. Braunstein and P. van Loock, Quantum information with continuous variables, Rev. Mod. Phys. 77, 513 (2005).

[6] S. L. Braunstein and H. J. Kimble, Teleportation of Continuous Quantum Variables, Phys. Rev. Lett. 80, 869 (1998).

[7] T. Opatrný and G. Kurizki, Matter-Wave Entanglement and Teleportation by Molecular Dissociation and Collisions, Phys. Rev. Lett. 86, 3180 (2001).

[8] K. Hammerer, A. S. Sørensen, and E. S. Polzik, Quantum interface between light and atomic ensembles, Rev. Mod. Phys. 82, 1041 (2010).

[9] V. Giovannetti, S. Lloyd, and L. Maccone, Quantum-enhanced measurements: Beating the standard quantum limit, Science 306, 1330 (2004).

[10] P. Mohn, Magnetism in the Solid State: An Introduction (Springer-Verlag, Berlin, 2006).
[11] J. B. Goodenough, Localized to Itinerant Electronic Transition in Perovskite Oxides (Springer-Verlag, Berlin, 2020).

[12] C. Fabre and N. Treps, Modes and states in quantum optics, Rev. Mod. Phys. 92, 035005 (2020).

[13] Entropy of entanglement $E_{\psi^{A B}}$ of a pure bipartite state $\left|\psi^{A B}\right\rangle$ is defined as the von Neumann entropy of the reduced density operator of one of the two subsystems $A$ or $B$. Explicitly, given the Schmidt form $\left|\psi^{A B}\right\rangle=\sum_{n} c_{n}\left|\varphi_{n}^{A}\right\rangle\left|\varphi_{n}^{B}\right\rangle$, one has $E_{\psi^{A B}}=$ $-\sum_{n}\left|c_{n}\right|^{2} \log _{2}\left|c_{n}\right|^{2}$.

[14] X. Zhu, A. Edström, and C. Ederer, Magnetic exchange interactions in $\mathrm{SrMnO}_{3}$, Phys. Rev. B 101, 064401 (2020).

[15] S. Meyer, B. Dupé, P. Ferriani, and S. Heinze, DzyaloshinskiiMoriya interaction at an antiferromagnetic interface: Firstprinciples study of Fe/Ir bilayers on Rh(001), Phys. Rev. B 96, 094408 (2017).

[16] G.-W. Chern, C. J. Fennie, and O. Tchernyshyov, Broken parity and a chiral ground state in the frustrated magnet $\mathrm{CdCr}_{2} \mathrm{O}_{4}$, Phys. Rev. B 74, 060405(R) (2006).

[17] K. Zakeri, Probing of the interfacial Heisenberg and Dzyaloshinskii-Moriya exchange interaction by magnon spectroscopy, J. Phys.: Condens. Matter 29, 013001 (2017).

[18] P. Zanardi, D. A. Lidar and S. Lloyd, Quantum Tensor Product Structures are Observable Induced, Phys. Rev. Lett. 92, 060402 (2004).

[19] D. Bossini, S. Dal Conte, G. Cerullo, O. Gomonay, R. V. Pisarev, M. Borovsak, D. Mihailovic, J. Sinova, J. H. Mentink, Th. Rasing, and A. V. Kimel, Laser-driven quantum magnonics and terahertz dynamics of the order parameter in antiferromagnets, Phys. Rev. B 100, 024428 (2019). 
[20] Dimensionless position and momentum quadratures of the $a_{\mathbf{k}}$ and $b_{\mathbf{k}}$ modes are $X_{\mathbf{k}}^{A}=\frac{a_{\mathbf{k}}+a_{\mathbf{k}}^{\dagger}}{\sqrt{2}}, X_{\mathbf{k}}^{B}=\frac{b_{\mathbf{k}}+b_{\mathbf{k}}^{\dagger}}{\sqrt{2}}, P_{\mathbf{k}}^{A}=\frac{a_{\mathbf{k}}-a_{\mathbf{k}}^{\dagger}}{i \sqrt{2}}$, and $P_{\mathbf{k}}^{B}=\frac{b_{\mathbf{k}}-b_{\mathbf{k}}^{\dagger}}{i \sqrt{2}}$.

[21] C. Gross, H. Strobel, E. Nicklas, T. Zibold, N. Bar-Gill, G. Kurizki, and M. K. Oberthaler, Atomic homodyne detection of continuous-variable entangled twin-atom states, Nature $\mathbf{4 8 0}$, 219 (2011).

[22] J. Peise, I. Kruse, K. Lange, B. Lücke, L. Pezzè, J. Arlt, W. Ertmer, K. Hammerer, L. Santos, A. Smerzi, and C. Klempt, Satisfying the Einstein-Podolsky-Rosen criterion with massive particles, Nature Comm. 6, 8984 (2015).

[23] J. Li, Y. Liu, N. Huo, L. Cui, S. Feng, X. Li, and Z. Y. Ou, Measuring continuous-variable quantum entanglement with parametric-amplifier-assisted homodyne detection, Phys. Rev. A 101, 053801 (2020).

[24] H. Y. Yuan and X. R. Wang, Magnon-photon coupling in antiferromagnets, Appl. Phys. Lett. 110, 082403 (2017).

[25] D. Lachance-Quirion, S. P. Wolski, Y. Tabuchi, S. Kono, K. Usami, and Y. Nakamura, Entanglement-based single-shot detection of a single magnon with a superconducting qubit, Science 367, 425 (2020).
[26] A. Voigt and J. Richter, The $J_{1}-J_{2}$ antiferromagnet on the square lattice with Dzyaloshinskii-Moriya interaction: An exact diagonalization study, J. Phys.: Condens. Matter 8, 5059 (1996).

[27] G. Beutier, S. P. Collins, O. V. Dimitrova, V. E. Dmitrienko, M. I. Katsnelson, Y. O. Kvashnin, A. I. Lichtenstein, V. V. Mazurenko, A. G. A. Nisbet, E. N. Ovchinnikova, and D. Pincini, Band Filling Control of the Dzyaloshinskii-Moriya Interaction in Weakly Ferromagnetic Insulators, Phys. Rev. Lett. 119, 167201 (2017).

[28] J. Li, S.-Y. Zhu, and G. S. Agarwal, Magnon-Photon-Phonon Entanglement in Cavity Magnomechanics, Phys. Rev. Lett. 121, 203601 (2018).

[29] J. Li and S.-Y. Zhu, Entangling two magnon modes via magnetostrictive interaction, New J. Phys. 21, 085001 (2019).

[30] Z. Zhang, M. O. Scully, and G. S. Agarwal, Quantum entanglement between two magnon modes via Kerr nonlinearity driven far from equilibrium, Phys. Rev. Research 1, 023021 (2019).

[31] H. Y. Yuan, S. Zheng, Z. Ficek, Q. Y. He, and M.-H. Yung, Enhancement of magnon-magnon entanglement inside a cavity, Phys. Rev. B 101, 014419 (2020). 